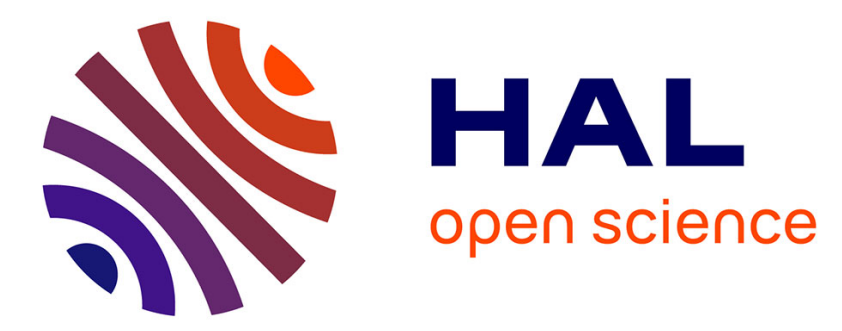

\title{
Maximizing Throughput of Linear Vehicular Ad-hoc NETworks (VANETs) - a Stochastic Approach
}

\author{
Bartlomiej Blaszczyszyn, Paul Mühlethaler, Yasser Toor
}

\section{To cite this version:}

Bartlomiej Blaszczyszyn, Paul Mühlethaler, Yasser Toor. Maximizing Throughput of Linear Vehicular Ad-hoc NETworks (VANETs) - a Stochastic Approach. European Wireless, May 2009, Aalborg, Denmark. 10.1109/EW.2009.5358011 . inria-00439793

\section{HAL Id: inria-00439793 https://hal.inria.fr/inria-00439793}

Submitted on 8 Dec 2009

HAL is a multi-disciplinary open access archive for the deposit and dissemination of scientific research documents, whether they are published or not. The documents may come from teaching and research institutions in France or abroad, or from public or private research centers.
L'archive ouverte pluridisciplinaire HAL, est destinée au dépôt et à la diffusion de documents scientifiques de niveau recherche, publiés ou non, émanant des établissements d'enseignement et de recherche français ou étrangers, des laboratoires publics ou privés. 


\section{Maximizing Throughput of Linear Vehicular Ad-hoc NETworks (VANETs) — a Stochastic Approach}

\author{
Barłomiej Błaszczyszyn \\ INRIA/ENS \\ Paris FRANCE \\ Bartek.Blaszczyszyn@ens.fr
}

\author{
Paul Mühlethaler \\ INRIA Rocquencourt \\ Le Chesnay FRANCE \\ Paul.Muhlethaler@inria.fr
}

\author{
Yasser Toor \\ INRIA Rocquencourt \\ Le Chesnay FRANCE \\ Yasser.Toor@inria.fr
}

\begin{abstract}
In this paper we use stochastic geometry to propose two models for Aloha-based linear VANETs. The first one uses Signal over Interference plus Noise Ratio (SINR) capture condition to qualify a successful transmission, while the second one expresses the transmission throughput as a function of SINR using Shannon's law. Assuming Poisson distribution of vehicles, power-law mean path-loss and Rayleigh fading, in these models we derive explicit formulas for the mean throughput and the probability of a successful reception at a given distance. Furthermore, we optimize two quantities directly linked to the achievable network throughput: the mean density of packet progress and the mean density of information transport. This is realized by tuning the communication range and the probability of channel access. We also present numerical examples and study the impact of external noise on an optimal tuning of network parameters.
\end{abstract}

Index Terms-VANETs, MAC (Medium Access Control) Layer Optimization, Throughput

\section{INTRODUCTION}

VANETs are important because they can help to increase safety on our road networks. However they are difficult to study through simulations because they can encompass a very large number of nodes which are usually highly mobile. Additionally, their node density can also vary rapidly. Consequently there has been increasing interest by the research community in analytical modeling of VANETs. Such an analysis would be of great use to design efficient and reliable VANETs.

Several current wireless communication networks use Carrier Sense Multiple Access (CSMA) or a variant as their MAC protocol. While CSMA is in common use, it is difficult to study analytically because it leads to complex patterns of nodes transmitting simultaneously. In contrast the Aloha protocol leads to simpler patterns, if we assume that the vehicle positions follow a spatial Poisson process.

Since Aloha is easier to study analytically than CSMA, we have chosen it as the MAC protocol for our linear VANET. We believe that the analysis presented in this paper will be of help to anyone studying the characteristics of VANETs and designing applications, protocols and hardware for them. In other words, we believe that this paper provides a solid insight into dependence of the network behavior on key parameters such as the node density, probability of medium access or typical communication range.

\section{RELATED WORK}

There have been several simulation studies of VANETs, in particular using CSMA-based MAC schemes. However, to the best of authors' knowledge, there are not many publications on the analytical study of linear VANETs with a solid interference model and SINR dependent performance metrics. A few analytical studies of CSMA techniques in two-dimensional (2D) MANET networks can be found such as [3], but with a very simplified interference models. For a precise interference model, the analysis of (slotted) Aloha is much easier as compared to a similar analysis of CSMA. However most of the studies of Aloha are for 2D MANET networks such as [5, 6].

The contribution of this paper is a rigorous analysis of some linear VANET models using (slotted) Aloha as their access scheme. The basic network and interference model, described in Section III-A, as well as the mathematical tools for its analysis are borrowed from [1] and [2]. Specifically, in Section III-B we analyze the network performance under a SINR capture (non-outage) condition, while in Section III-C we assume that the channel throughput is given by the famous Shannon's $\log (1+$ SINR $)$ law. In both cases, we optimize the global network throughput using the transmission range and Aloha medium access probability. In contrast to the $2 \mathrm{D}$ situation this optimization in 1D does not degenerate. We also study the impact of external noise on this optimization. Section IV discusses the numerical examples, followed by the conclusion in Section V.

\section{A STOCHASTIC MODEL FOR A LINEAR VANET}

\section{A. Network and Interference Model}

The model and the mathematical tools are borrowed from [1] and [2]. We consider an infinite linear network. Let $\Phi=$ $\left\{\left(X_{i}, e_{i}\right)\right\}$ be a marked Poisson point process with intensity $\lambda$ on the line $\mathbb{R}$, where

- $\Phi=\left\{X_{i}\right\}$ denotes the locations of vehicles,

- $\left\{e_{i}\right\}_{i}$ is the medium access indicator of station $i ; e_{i}=1$ for the station which is allowed to emit and $e_{i}=0$ for the station which is not allowed to emit. The random variables $e_{i}$ are independent, with $\mathbf{P}\left(e_{i}=1\right)=p$.

Note first that $\Phi$ can be represented as a pair of independent Poisson p.p. representing emitters $\Phi^{1}=\left\{X_{i}: e_{i}=1\right\}$, and nodes $\Phi^{0}=\left\{X_{i}: e_{i}=0\right\}$ which are not allowed to emit (at a given time slot). These processes have intensities of $\lambda p$ and $\lambda(1-p)$ respectively.

For simplicity we assume that each transmitting vehicle (in $\Phi^{1}$ ) uses the same transmit power $S$, with a default value of $S=1 \mathrm{~W}$. To compute the mean received power (without fading effects) we use the attenuation function $l(r)=(A r)^{-\beta}$ where $r$ is the distance between the emitter and the receiver. We assume that $A=1$ without loss of generality. Our mathematical linear model of the network requires $\beta>1$ (in order for the sum of all powers received at a given location to have a finite mean). Typically beta is larger than 2 and our default value is $\beta=4$. 
We also take into account a random fading $F_{(x, y)}$ between two vehicles located respectively at $x$ and $y$. Thus, the actual signal power decay between these two vehicles will be $F_{(x, y)} l(|x-y|)$. Throughout the paper we assume that the values of $F_{(x, y)}$ are independent and exponentially distributed identically with a mean $1 / \mu$, which corresponds to the situation of independent Rayleigh fading.

We also consider an independent external noise (i.e., independent of $\Phi$, e.g., thermal) and denote it at (a given location) by $W$.

We assume that each vehicle in $\Phi$ transmits towards its own receiver located within the distance $R$ from it (and not represented in $\Phi$ ). This is sometimes called the "bipolar network model" in contrast to the model where each emitter in $\Phi^{1}$ chooses its receiver within $\Phi^{0}$. It allows us to study essential network performance characteristics at the medium access level without modeling particular routing schemes.

Suppose that a vehicle located at $x$ transmits a signal with power $S$ that is received by a vehicle located at $y$. The Signal over Interference plus Noise Ratio (SINR) of this communication will be

$$
\operatorname{SINR}_{(x, y)}=\frac{S F_{(x, y)} l(|x-y|)}{W+I_{\Phi^{1}}(y)}
$$

where $I_{\Phi^{1}}$ is the shot-noise process of $\Phi^{1}: I_{\Phi^{1}}(y)=$ $\sum_{X_{i} \in \Phi^{1}} S F_{\left(y, X_{i}\right)} l\left(\left|y-X_{i}\right|\right)$.

In what follows we will present the analysis of the network performance assuming first some particular coding scheme that requires SINR to be larger than some threshold $T$ (SINR capture (non-outage) condition) for the successful transmission at a fixed given bit-rate. Later, in subsection III-C, we will assume an adaptive coding scheme in which, for a given SINR level, the appropriate choice of the coding scheme allows us to obtain a bit-rate close to that given by Shannon's law.

\section{B. SINR capture}

In this section we assume a fixed given bit-rate and that $y$ successfully receives the signal form $x$ if

$$
\operatorname{SINR}_{(x, y)} \geq T,
$$

where $\operatorname{SINR}_{(x, y)}$ is given by (3.1) and $T$ is the SINR-threshold related to the bit-rate given some particular coding scheme.

Let us denote the indicator that (3.2) holds by $\delta\left(x, y, \Phi^{1}\right)$. Note that by stationarity of $\Phi^{1}$, the probability $\mathbf{E}\left[\delta\left(x, y, \Phi^{1}\right)\right]$ depends only on the distance $x-y$ and not on the specific locations of $(x, y)$; so we can define the probability of reception $p_{|x-y|}(\lambda p)=\mathbf{E}\left[\delta\left(x, y, \Phi^{1}\right)\right]$, where $\lambda p$ is the intensity of the emitters $\Phi^{1}$. Note also that this probability depends on $\lambda$ and $p$ only through their product $\lambda p$ and thus it is enough to find its expression for $p=1$. The following result is crucial for the whole of our network analysis. It is inspired by the analogous result obtained for 2D networks in [1].

Proposition 3.1: Assume $p=1$. The probability of the successful transmission is equal to

$$
p_{R}(\lambda)=\exp \left\{-\frac{2 \pi \lambda R T^{\frac{1}{\beta}}}{\beta \sin (\pi / \beta)}\right\} \psi_{W}(\mu T / l(R))
$$

where $\psi_{W}(\xi)=\mathbf{E}\left[e^{-\xi W}\right]$ is the Laplace transform of the noise $W$.

Proof: The proof goes along the same lines as given for the $2 \mathrm{D}$ case in [1]. Specifically,

$$
\begin{aligned}
p_{R}(\lambda) & =\mathbf{P}\left(F S \geq T\left(W+I_{\Phi}\right) / l(R)\right) \\
& =\int_{0}^{\infty} e^{-\mu s T / l(R)} \mathrm{d} \operatorname{Pr}\left(W+I_{\Phi} \leq s\right) \\
& =\psi_{I_{\phi}}(\mu T / l(R)) \psi_{W}(\mu T / l(R)),
\end{aligned}
$$

where $\psi_{I_{\phi}}$ denotes the Laplace transform of the Poisson shot noise. It is known that (it can be derived from the formula for the Laplace functional of the Poisson p.p. (see e.g. [4, p. 61]) and was already used in 2D in [1]).

$$
\psi_{I_{\Phi}}(\xi)=\exp \left\{-2 \lambda \int_{0}^{\infty} 1-\mathbf{E}\left[e^{-\xi S F l(|x|)}\right] \mathrm{d} x\right\} .
$$

Introducing the exponential distribution of the fading and $S=1$ we obtain:

$$
\begin{aligned}
\psi_{I_{\Phi}}(\xi) & =\exp \left\{-2 \lambda \int_{0}^{\infty} 1-\int_{0}^{\infty} \mu e^{-\xi u l(|x|)} e^{-\mu u} \mathrm{~d} u \mathrm{~d} x\right\} \\
& =\exp \left\{-\frac{2 \pi \lambda \xi^{1 / \beta}}{\mu^{1 / \beta} \beta \sin (\pi / \beta)}\right\} .
\end{aligned}
$$

Consequently,

$$
\psi_{I_{\Phi}}(\mu T / l(R))=\exp \left\{-\frac{2 \pi \lambda R T^{\frac{1}{\beta}}}{\beta \sin (\pi / \beta)}\right\} .
$$

This concludes the proof.

Note that if $W=0$ then $\psi_{W}(\xi) \equiv 1$ and the formula for the successful reception probability simplifies to

$$
p_{R}(\lambda)=\exp \left\{-\frac{2 \pi \lambda R T^{\frac{1}{\beta}}}{\beta \sin (\pi / \beta)}\right\} .
$$

In the remaining part of this section we will consider a general medium access probability $0 \leq p \leq 1$. Recall, in this case the corresponding reception probability is equal to $p_{R}(p \lambda)$.

Using Campbell's formula (see [8]) we can express the mean total number of successful transmissions per unit length of the network (the density of successful transmissions) by $\lambda p p_{R}(\lambda p)$. Moreover, the mean progress of the typical transmission is simply equal to $R p_{R}(\lambda p)$.

In the remaining part of this section we will be mainly interested in the mean density of progress $d_{\text {prog }}$, defined as the expected total progress of all the transmissions per unit length of the network and per time slot. Again, by Campbell's formula, it can be expressed by $d_{\text {prog }}(R, \lambda, p)=\lambda p R p_{R}(\lambda p)$. This metric is interesting because it is directly linked to the network throughput i.e., number of bit-meters transmitted per unit length of the network and per unit of time.

In the following result we optimize this metric in $p$. Let us denote a critical communication range by

$$
R^{*}=\frac{\beta \sin (\pi / \beta)}{2 \pi T^{\frac{1}{\beta}} \lambda} .
$$

Proposition 3.2: If $R \geq R^{*}$ then the value of $p$ that 
maximizes the mean density of progress $d_{\text {prog }}(R, \lambda, p)$ is given by

$$
p^{*}=\frac{\beta \sin (\pi / \beta)}{2 \pi T^{\frac{1}{\beta}} \lambda R}=R^{*} / R
$$

and the maximum value is equal to

$$
d_{\text {prog }}\left(R, \lambda, p^{*}\right)=\frac{\beta \sin (\pi / \beta)}{2 \pi e T^{\frac{1}{\beta}}} \psi_{W}\left(\mu T R^{\beta}\right) .
$$

If $R \leq R^{*}$ then $p^{*}=1$ and

$$
d_{\text {prog }}\left(R, \lambda, p^{*}\right)=\lambda R \exp \left\{-\frac{2 \pi \lambda R T^{\frac{1}{\beta}}}{\beta \sin (\pi / \beta)}\right\} \psi_{W}\left(\mu T R^{\beta}\right) .
$$

Proof: The result follows from Proposition 3.1 by differentiating the explicit formula for mean density of progress with respect to $p$.

We now consider the optimization of the mean density of progress jointly in $p$ and $R$.

Proposition 3.3: If $W>0$ (with non-null probability) then the maximum (in $p$ and $R$ ) of the mean density of progress $d_{\text {prog }}(R, \lambda, p)$ is equal to

$$
\max _{R \in\left[0, R^{*}\right]}\left\{\lambda R \exp \left\{-\frac{2 \pi \lambda R T^{\frac{1}{\beta}}}{\beta \sin (\pi / \beta)}\right\} \psi_{W}\left(\mu T R^{\beta}\right)\right\}
$$

and is attained for $p^{*}=1$ and an $R$ that maximizes the expression in (3.5). In the absence of noise ( $W \equiv 0$ ) the maximal mean density of progress $d_{\text {prog }}(R, \lambda, p)$ is equal to

$$
\beta \sin (\pi / \beta) /\left(2 e \pi T^{\frac{1}{\beta}}\right)
$$

and is attained whenever $p R=R^{*}$ with $R \geq R^{*}$.

Proof: The result follows directly from Proposition 3.2. If $W>0$ then $\psi_{W}\left(\mu T R^{\beta}\right)$ is strictly decreasing function of $R$ and the maximum of (3.3) with $R \geq R^{*}$ is attained for $R=R^{*}$. Moreover, the value of (3.4) with $R=R^{*}$ is equal to the value of (3.3) with $R=R^{*}$. Consequently the maximum is attained for some $R \leq R^{*}$ and thus $p^{*}=1$. If we assume now that $W=0$, then $\psi_{W} \equiv 1$. It is then easy to show that the maximum of (3.4) on the interval $R \leq R^{*}$ is attained for $R=R^{*}$ and is equal to the value of (3.3) with $\psi_{W} \equiv 1$. Consequently the optimal choice of $p$ and $R$ is $R \geq R^{*}$ and $p=R^{*} / R$. This completes the proof.

We will make a few remarks regarding the result presented in Proposition 3.3.

Remarks: 1) Our linear VANET model can be optimized (with respect to the mean density of progress) jointly in the communication range $R$ and in the medium access probability $p$. This is in contrast to the 2D situation considered in [1]. In this latter case the maximum in $p$ of the mean density of progress is of order $O(1 / R)$ and joint optimization in $p$ and $R$ is attained at $R=0$ and $p=1$, in which case the density of progress is equal to $\infty$. This solution is of course not acceptable from a practical point of view. The difference between linear networks and 2D networks comes from the fact that in the latter, a transmission "consumes" an area of order $O\left(R^{2}\right)$ and the progress is equal to $R$ - a situation that leads to the optimal network consisting of dense packing of small-range communications. In linear networks a transmission "consumes" a length of $O(R)$ and the progress is also $R$. Thus the optimization of the density of progress does not degenerate.

2) If the external noise is not negligible $(W>0)$ then the best choice of VANET parameters requires some optimal communication range $R \leq R^{*}$ but nog access control $\left(p^{*}=1\right)$. Note that $R^{*} \leq 1 /\left(2 T^{1 / \beta} \lambda\right)$ and thus the optimal choice of $R$ may be smaller than $1 / \lambda$ i.e., the mean distance between two points of $\Phi$, especially when $T$ is not too small as it is the case in the absence of sophisticated interference cancellation techniques like spreading or CDMA. (For example, for $\beta=4$ and $T=1, R^{*}$ is $1 / 4$ th of the mean inter-point distance.) There is some concern about the pertinence of such a network model. In reality the receiver is chosen by the routing scheme among the neighbors of the emitter and thus a reasonable choice of $R$ should be at least of order $1 / \lambda$. This might suggest a protocol closer to that of a "delay tolerant network" (that is transmit only when neighboring vehicles are sufficiently close).

$3)$ If the external noise is negligible $(W=0)$ then the VANET network designer is allowed to choose arbitrarily $R \geq R^{*}$ and tune the medium access parameter $p^{*}=R^{*} / R$ correspondingly. The numerical examples considered in Section IV suggest that the noise of order of $W=10^{-10} \mathrm{~mW}$ and smaller can be neglected, while $W=10^{-6} \mathrm{~mW}$ cannot. ${ }^{1}$

\section{Optimal Adaptive Coding}

In Section III-B we have assumed that a transmission (of a given bit-rate) either fails or is successful and this latter holds true if the SINR is above some given threshold $T$. In this section we consider a situation in which for any value of SINR some communication is feasible with the bit-rate $\tau$ that depends on this value of SINR. This assumption corresponds to an adaptive coding in the channel: if the SINR is high, the coding can be 'loose' and thus the bit-rate is high, whereas with a small SINR the coding must be 'tight' and thus the throughput is low.

Link adaptations and turbo codes permit us to approach the well known theoretical maximal-bit rate of the Gaussian channel (AWGN) $B \log (1+\mathrm{SINR})$ where $B$ is the channel bandwidth ${ }^{2}$. Inspired by Shannon's law, and assuming for simplicity that $B=1$, we now say in our VANET model that the vehicle at $y$ receives the signal from the vehicle at $x$ with the throughput (bit-rate) given by

$$
D\left(x, y, \Phi^{1}\right)=\log \left(1+\operatorname{SINR}_{(x, y)}\right),
$$

where $\operatorname{SINR}_{(x, y)}$ is given by (3.1). Note again that by stationarity of $\Phi^{1}$ the mean throughput

$$
\tau(R, \lambda p)=\mathbf{E}\left[D\left(x, y, \Phi^{1}\right)\right]
$$

depends only on the distance $|x-y|=R$ and not on the specific locations of $(x, y)$; recall that $\lambda p$ is the intensity of the emitters $\Phi^{1}$. We can now prove the following basic result for our VANET model with adaptive coding.

\footnotetext{
${ }^{1}$ A recent study [7] of vehicle-to-vehicle wireless channels suggests the noise order of magnitude $10^{-10.27} \mathrm{~mW}$.

${ }^{2}$ With the loss of a few $\mathrm{dB}$ in SINR
} 
Proposition 3.4: Assume $p=1$. The mean throughput is equal to

$$
\tau(R, \lambda)=\beta \int_{0}^{\infty} \exp \left\{\frac{-2 \pi \lambda R v}{\beta \sin (\pi / \beta)}\right\} \frac{v^{\beta-1}}{1+v^{\beta}} \psi_{W}\left(\mu R^{\beta} v\right) d v .
$$

Proof: The proof goes along the same lines as given for the $2 \mathrm{D}$ case in [2]. First note that

$$
\mathbf{E}[\log (1+\operatorname{SINR})]=\int_{0}^{\infty} \mathbf{P}\{\log (1+\operatorname{SINR})>t\} \mathrm{d} t .
$$

Substituting,

$\mathbf{P}\{\log (1+\operatorname{SINR})>t\}=\mathbf{P}\left\{\operatorname{SINR}>e^{t}-1\right\}=p_{R}\left(\lambda, e^{t}-1\right)$,

where we introduce into the previous notation of $p_{R}$ the explicit dependence on $T=e^{t}-1$, and obtain

$$
\tau(R, \lambda)=\int_{0}^{\infty} p_{R}\left(\lambda, e^{t}-1\right) \mathrm{d} t
$$

Using Proposition 3.1 and substituting $\left(e^{t}-1\right)^{1 / \beta}=v$ the expected result is obtained.

We can now define an important metric; analogous to the mean density of progress considered in the previous section. We will call the mean density of transport $d_{\text {trans }}$ the expected number of bit-meters transported by the unit length of the network per unit of time. By Campbell's formula it can be expressed in our network as

$$
d_{\text {trans }}(R, \lambda, p)=R \lambda p \tau(R, \lambda p)
$$

Recall that this metric is related to the achievable network throughput under the second model (based on Shannon's law).

In what follows, we characterize the choice of the network parameters $R$ and $p$ that maximize $d_{\text {trans }}$. Using the result of Proposition 3.4 it can be shown that $R \tau(R, \lambda)$ converges to 0 when $R \rightarrow 0$, as well as, when $R \rightarrow \infty$. We conjecture that:

(C) $R \tau(R, \lambda)$ with $W \equiv 0$ admits one global maximum for $R=Y^{*}$ and is strictly increasing for $R<Y^{*}$.

By Proposition 3.4 this critical (in the absence of noise) communication range $Y^{*}$ can be characterized as the solution of the following equation

$$
\begin{aligned}
& \int_{0}^{\infty} \exp \left\{\frac{-2 \pi \lambda Y^{*} v}{\beta \sin (\pi / \beta)}\right\} \frac{v^{\beta-1}}{1+v^{\beta}} \mathrm{d} v \\
& \quad=\frac{2 \pi \lambda Y^{*}}{\beta \sin (\pi / \beta)} \int_{0}^{\infty} \exp \left\{\frac{-2 \pi \lambda Y^{*} v}{\beta \sin (\pi / \beta)}\right\} \frac{v^{\beta}}{1+v^{\beta}} \mathrm{d} v .
\end{aligned}
$$

The following result is similar to Proposition 3.3.

Proposition 3.5: Assume that condition (C) is satisfied. In the absence of noise ( $W \equiv 0)$ the maximal mean density of transport $d_{\text {trans }}$ is attained whenever $p R=Y^{*}$ with $R \geq Y^{*}$. If $W>0$ (with non-null probability) then the maximum (in $p$ and $R$ ) of the mean density of transport $d_{\text {trans }}$ is equal to

$$
\max _{R \in\left[0, Y^{*}\right]} \beta \int_{0}^{\infty} \exp \left\{\frac{-2 \pi \lambda R v}{\beta \sin (\pi / \beta)}\right\} \frac{v^{\beta-1}}{1+v^{\beta}} \psi_{W}\left(\mu R^{\beta} v^{\beta}\right) d v
$$

and is attained for $p^{*}=1$ and an $R$ that maximizes (3.7).

Proof: Note first by Proposition 3.4 that if $W \equiv 0$ then $d_{\text {trans }}(R, \lambda, p)$ depends on $p$ and $R$ only through the product $p R$. This and the definition of $Y^{*}$ proves the first part of the result. Assume now that $W>0$. Then $\psi_{W}\left(\mu R^{\beta} \nu^{\beta}\right)$ is strictly decreasing in $R$ and thus the maximum of $d_{\text {trans }}(R, \lambda, p)$ is attained for some $R \leq Y^{*}$. By assuming that $R \tau(R, \lambda)$ with $W \equiv 0$ is strictly increasing for $R<Y^{*}$ we conclude that $p^{*}=1$.

\section{NUMERICAL EXAMPLES}

In this section we give some numerical examples; in particular showing the impact of noise $W$ on the network design.

Throughout this section we assume that the density of the network is $\lambda=0.01$ (vehicles per $1 \mathrm{~m}$ of the network, i.e., 10 per $1 \mathrm{~km})$, the exponential fading with mean $1 / \mu=1$ and path-loss exponent $\beta=4$.

We use the result of Proposition 3.1 to compute the mean density of progress $d_{\text {prog }}$ for $T=10$ and different values of noise $W$, transmission range $R$ and function of the transmission probability $p$. The results of these computations, carried out with Maple, are given in Figure 1. We can verify the result of Proposition 3.3 with $W \equiv 0 \mathrm{~mW}$; the optimum density of progress is reached for $p R=R^{*} \approx 25.31$. We see that the noise $W=10^{-10} \mathrm{~mW}$ can be ignored. Indeed, the "true" maximum of the density of progress 0.093 is attained at $p=1$ and $R=$ 25.6. However, for $R=100$ and $p=0.25$ (yielding $p R=$ $25 \approx R^{*}$ ) the value of the density of progress is 0.085 which is still not far from the optimal value. However, in order to maximize the network performance with $W=10^{-6} \mathrm{~mW}$ it is necessary to tune the parameters close to the "true" optimum obtained for $p^{*}=1$ and $R=11.31$.

Similar observations can be made on Figure 2 that presents the mean density of transport (in the case of adaptive coding) evaluated using Proposition 3.4. In particular, in the absence of the external noise, the optimum density of transport value 0.53 is reached for $p R=Y^{*} \approx 21.7$. There is no visible difference when the noise is equal $W=10^{-10} \mathrm{~mW}$. The maximum 0.53 is attained for $p=1$ and $R=21.7$, however for $p=0.26$ and $R=100$ the value of the mean density of transport is still 0.5 . So noise of order $W=10^{-10} \mathrm{~mW}$ can be ignored. However, when $W=10^{-6} \mathrm{~mW}$, the maximum 0.28 is reached when $p=1$ and $R=8.9$. To maximize the network performance with $W=10^{-6} \mathrm{~mW}$ the parameters must be tuned close to this "true" optimum.

Similar optimizations of the mean density of progress and the mean density of transport with other values of $p, R, W, \beta$, $\mu$ and $S$ can easily be carried out.

\section{CONCLUSiON}

VANETs are an important type of MANET because they can help to increase road safety. In this paper we have tried to further our understanding of them by making a stochastic analysis of an Aloha-based infinite linear VANET. We propose two models based on SINR capture condition and Shannon's law, respectively. Using these models we show how we can maximize mean packet progress and mean density of information transport by optimizing the transmission probability and the transmission range. We also take into account random fading between transmitting and receiving vehicles as well 

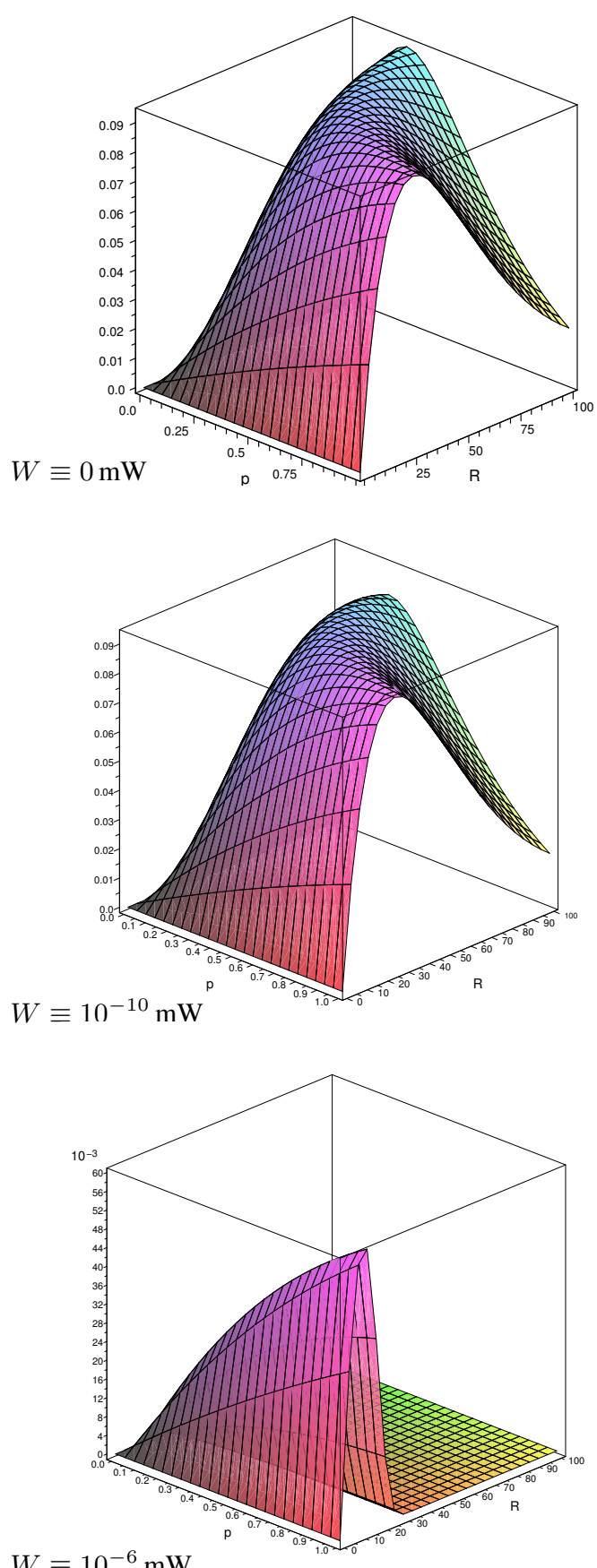

$$
W \equiv 10^{-6} \mathrm{~mW}
$$

Fig. 1. Mean density of progress versus transmission probability $p$ and transmission range $R$ for three different values of the external noise power $W$.w

as independent external noise. Our results reveal interesting dependencies between the performance of the network and its parameters. These dependencies are intrinsic to 1D scenarios usually assumed for VANETs. They highlight the difference between 1D networks used to model VANETs and 2D networks typically used for MANETs.

\section{REFERENCES}

[1] F. Baccelli, B. Blaszczyszyn, and P. Mühlethaler. An Aloha Protocol for Multihop Mobile Wireless Networks. IEEE Transactions on Information Theory, 52(2):421-436, 2006.

[2] F. Baccelli, B. Błaszczyszyn, and P. Mühlethaler. Stochastic analysis of spatial and opportunistic Aloha. IEEE JSAC, special issue on Stochastic Geometry and Random Graphs for Wireless Networks, 2009. accepted.
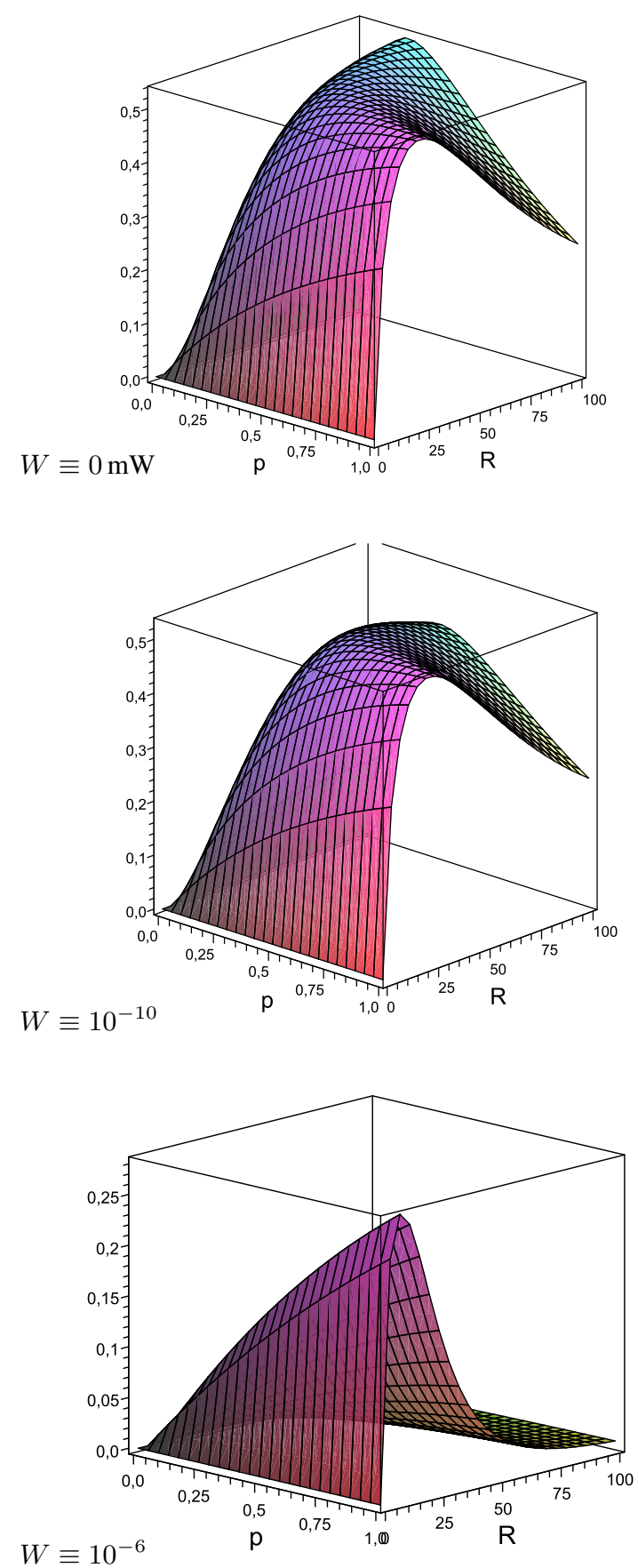

Fig. 2. Mean density of transport versus transmission probability $p$ and transmission range for three different values of the external noise power $W$.

[3] Y. Chen, Q. Zeng, and D. Agrawal. Analytical modeling of MAC protocol in ad hoc networks. Wireless Communications and Mobile Computing, pages 45-59, 2006.

[4] D. J. Daley and D. Vere-Jones. An Introduction to the Theory of Point Processes, vol. 2. Springer, 2008. second edition.

[5] N. Ehsan and R. Cruz. On the Optimal SINR in Random Access Networks with Spatial Reuse. In Proc. of the CISS 2006 Conference, Princeton University, NJ, USA., 2006.

[6] A. Hunter, J. Andrews, and S. Weber. Capacity Scaling of Ad Hoc Networks with Spatial Reuse. In submitted for publication, 2008.

[7] Alexander Paier, Johan Karedal, Nicolai Czink, Charlotte Dumard, Thomas Zemen, Fredrik Tufvesson, Andreas F. Molisch, and Christoph F. Mecklenbräuker. Characterization of vehicle-to-vehicle radio channels from measurements at $5.2 \mathrm{ghz}$. Wireless Pers Commun, 10.1007/s11277-0089546-6, 2008.

[8] D. Stoyan, W. Kendall, and J. Mecke. Stochastic Geometry and its Applications. Wiley, Chichester, 1995. 